\title{
TRON - Hardware-in-the-Loop Test Facility for Lunar Descent and Landing Optical Navigation
}

\author{
Hans Krüger* Stephan Theil ** \\ * GNC Systems Department, German Aerospace Center, Bremen, \\ 28359 Germany (e-mail: hans.krueger@dlr.de). \\ ** GNC Systems Department, German Aerospace Center, Bremen, \\ 28359 Germany, (e-mail: stephan.theil@dlr.de)
}

\begin{abstract}
Future exploration missions require advanced optical sensors for precise navigation and landing site evaluation. The Testbed for Robotic Optical Navigation (TRON) is a Hardwarein-the-Loop test environment, with the purpose to support the development of optical navigation technology, and to qualify breadboards to TRL 4, and to qualify flight models to TRL 5-6. In this paper the design and ongoing realization of TRON is discussed. The first application of TRON is to simulate relevant parts of the lunar landing. After illustrating the concept, the building blocks of the laboratory are explained in detail. These are the simulation of the scaled dynamics via a $7-\mathrm{DOF}$ robot, the simulation of the optical environment via a black out system and a lighting system, and the simulation of the terrain geometry via scaled 3D terrain models. With modifications TRON can also provide relevant environments for Mars, asteroids and moons.
\end{abstract}

Keywords: Hardware-in-the-loop test, optical navigation, safe and precise landing, spacecraft autonomy

\section{INTRODUCTION}

\section{DESIGN}

\subsection{Requirements and constraints}

The main goal of TRON is to provide the environment to qualify optical navigation sensor breadboards to TRL 4 and to qualify flight hardware to TRL 6. Consequently TRON shall provide a hardware-in-the-loop test capability for these kind of sensors. Thus the optical, geometric and dynamic conditions of a lunar landing shall be simulated in the testbed.

The typical mission profile of a precise and safe lunar landing consists of different phases which are briefly explained in the following. After the lunar orbit injection the spacecraft is typically situated in a circular Lunar orbit with altitudes in the range of 100 to $110 \mathrm{~km}$. At a suitable point the lander enters the descent orbit (DO), where a Hohmann transfer down to an periselenium between $\approx 10$ $\mathrm{km}$ and $15 \mathrm{~km}$ is performed. Near the periselenium of the DO the powered descent (PD) is initiated. The PD covers a distance of several hundred kilometers and ends at an altitude of $1-2 \mathrm{~km}$, where most of the orbital speed is reduced. At this point the lander approaches high gate, gaining visibility of landing site. The evaluation of the landing area is starting at low gate position at around $1 \mathrm{~km}$. This is also called hazard avoidance (HA) or hazard recognition. During the further approach a safe landing site should be selected and then terrain relative navigation (TRN) techniques are performed to reach this location. There are various examples in literature for this kind of mission profile, e.g. the Apollo Program (Loucks et al. (2005)), the Vision for Space Exploration (Sostaric (2007)) or the ESA Next lunar lander (Neveu et al. (2010)). 


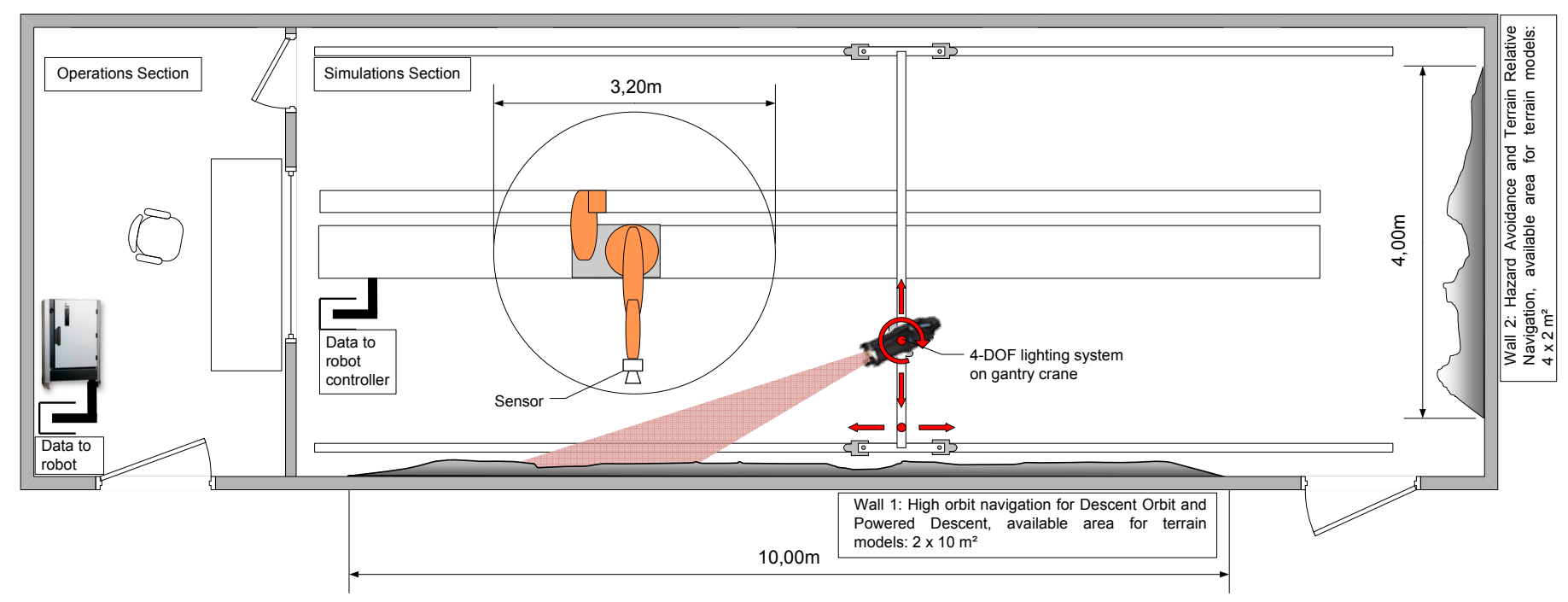

Fig. 1. Schematic top view of TRON

As stated in section 1, there are different approaches in development, which aim to apply optical navigation techniques during all landing phases. To test this variety of sensors TRON shall provide a simulation of all the phases of lunar landing.

Future missions like the Next Lunar Lander (Houdou (2008)) require a precise landing in the order of hundreds of meters and the selection of a safe landing site by avoiding obstacles bigger than $0.5 \mathrm{~m}$. Consequently the navigation requirements are in the order of $100 \mathrm{~m}$ until the approach of the landing site. Once a landing site is selected the requirement drops to the order of $1 \mathrm{~m}$ for successfully placing the lander in a safe area. The terrain models installed in the laboratory serve as the reference for the navigation sensors and must have at least this level of detail. Similarly, the device positioning the sensor dynamically over the terrain must have a precision better than the navigation requirements.

The minimum level of detail is constrained by the requirements of the Landing site evaluation, hence $\approx 0.5$ $\mathrm{m}$. Due to manufacturing limits the maximum possible resolution of the terrain model is $0.2 \mathrm{~mm}$ (see section 4 ), resulting in a scaling of 1:2500. The ground-projected range from DO until the landing is in the order of half a lunar circumference, $\approx 5500 \mathrm{~km}$. The height range is $100 \mathrm{~km}$. Covering the whole mission profile continuously would result in a laboratory space of $2000 \mathrm{~m}$ length and $40 \mathrm{~m}$ height. On the other hand the available space for the testbed in DLR Bremen covers $16 \mathrm{~m}$ length, $5 \mathrm{~m}$ width and $3 \mathrm{~m}$ height.

\subsection{Concept}

The requirements and constraints from section 2.1 led us to the following design decisions. The simulation of the lunar landing mission profile is performed in sections with suitable scaling factors applied for each section. For the representation of the lunar terrain we chose the application of scaled 3D models. They permit the test of 3D imaging sensors like stereo cameras and lidars. Furthermore the 3D models will be combined with a suitable lighting system to achieve high quality shadows in real time. In this way also for passive camera sensors a relevant optical environment can be achieved. Consequently, a light source with a color temperature near the sun's value shall be used. The light shall be placed on a movable device to provide variable solar irradiation angles. To avoid secondary lighting the walls and the ceiling have been completely covered with black fabric. Additionally the floor is covered with black carpet.

For the dynamic part of the simulation a 7-DOF system comprising a 6-DOF industrial robot on a rail was chosen. It is installed along the long axis of the room. The robot shall be controlled by a real-time (RT) computer system, which will also be responsible for the real world simulation of the space craft. Besides the simulation requirements some safety issues had to be addressed. During automatic robot action no human presence in its vicinity is allowed. As a result the laboratory was divided into a minor part for the operators and the major part for the simulation environment.

\subsection{Simulation options in TRON}

In this section the layout and the resulting options for simulations shall be explained. The robot is installed on a rail along the long centerline of the laboratory. The sensor to be qualified is installed at the robots tool center point (TCP). During the design process it has been found suitable to place the terrain models at two walls of the room. The resulting layout is illustrated in Fig. 1.

The wall at the long side provides a space of $2 \times 10 \mathrm{~m}^{2}$. It is foreseen for the simulation of the descent orbit (DO) and the powered descent (PD). The TCP can be moved along the entire terrain models placed at wall 1 , with a variable distance between $1 \mathrm{~m}$ and $2 \mathrm{~m}$. Considering a terrain model for the DO in a scale of 1:50000, the SC positions could be simulated over a ground distance of $500 \mathrm{~km}$ (equivalent to an orbit angle of $\approx 16$ degrees) and altitudes between 100 $\mathrm{km}$ and $50 \mathrm{~km}$. By choosing 1:10000, the late stage of the DO until the initiation of the PD could be simulated over altitudes from $10 \mathrm{~km}$ to $20 \mathrm{~km}$.

If a qualification of the whole DO is necessary it can be approximated by the combination of sections with different 


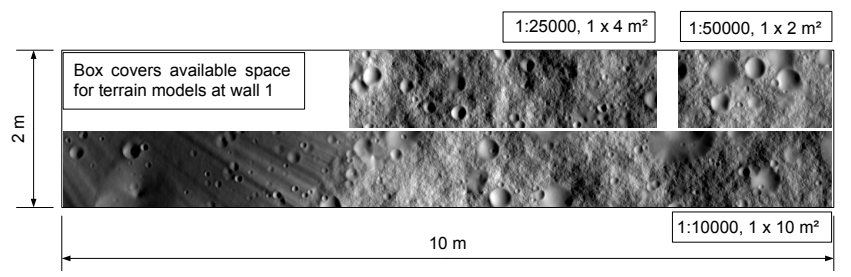

Fig. 2. Simulation of the descent orbit in 3 sections with different scales and terrain model sizes, providing a real-time simulation duration of $60 \mathrm{~s}$ for each

scales. While the orbital speed keeps nearly constant over the DO, the laboratory speed is dependent on the scale. This results in different simulation times for section of same dimensions but different scales. In Figure 2 it is shown how a constant simulation time of 1 min could be achieved by the suitable combination of scales and dimensions.

For the simulation of the $\mathrm{PD}$ a low scaling factor is necessary to achieve altitudes between $1 \mathrm{~km}$ and $10 \mathrm{~km}$. These cause high speed values of the TCP $>30 \mathrm{~cm} / \mathrm{s}$, resulting in short real-time simulation periods. Thus we consider preferably late stages in the PD for simulation, because the orbital speed is already reduced significantly. At an altitude of $5 \mathrm{~km}$ the speed is in the order of 600 $\mathrm{m} / \mathrm{s}$. With a scale of $1: 2500, v_{S C}=600 \mathrm{~m} / \mathrm{s}$ translates into $v_{l a b}=24 \mathrm{~cm} / \mathrm{s}$. Due to continuous braking in PD, a simulation time of $1 \mathrm{~min}$ could be realized by using a terrain model with a length of $\approx 9 \mathrm{~m}$.

The layout of wall 1 is under ongoing definition and will depend on further development steps of the ATON project.

Wall 2 is foreseen for the simulation of the last phase of the landing. The terrain models for this wall are also to be manufactured in 3D to serve as a sensor target for 3D imaging sensors. In this way the terrain relative navigation with respect to the landing site and the evaluation for safe areas can be tested hardware-in-the-loop. The TCP can be moved from a distance of $11 \mathrm{~m}$ to a distance of $1 \mathrm{~m}$ to the terrain model installed at wall 2 . The available space for terrain models has a width of $4 \mathrm{~m}$ and a height of $2 \mathrm{~m}$. The TCP can be moved with a radius of $\approx 1 \mathrm{~m}$ perpendicular to the rail.

By choosing a scale of 1:100 an approach from an altitude of $1100 \mathrm{~m}$ down to $100 \mathrm{~m}$ can be realized. During its approach the SC, i.e. the sensor system, can make 200 $\mathrm{m}$ of lateral movements. This restricts the simulation in terms of possible landing site dispersion and re-targeting abilities. With a higher scale of 1:200 the lateral movement could be increased to $400 \mathrm{~m}$. But it has to be considered that the error of a lidar increases with the scale. Assuming a constant real world error of $\pm 3 \mathrm{~mm}$, the simulated error doubles from $\pm 0.3 \mathrm{~m}(1: 100)$ to $\pm 0.6 \mathrm{~m}$ (1:200), which is in the order of the maximum allowed hazard size. A trade-off has to be performed between the necessary lateral movement and the acceptable errors for the individual instrument.

\section{DYNAMICS}

The task of the dynamic system is to realize a real-time controllable movement of the sensor system following the scaled mission profiles. The system has to be able to move
Table 2. Navigation requirements in lab scale

\begin{tabular}{cccc}
\hline & nav. req. & scale & nav. req. in lab dim. \\
\hline DO & $100 \mathrm{~m}$ & $1: 50000$ & $2 \mathrm{~mm}$ \\
DO and PD & $100 \mathrm{~m}$ & $1: 10000$ & $10 \mathrm{~mm}$ \\
HA and TRN & $1 \mathrm{~m}$ & $1: 200$ & $5 \mathrm{~mm}$ \\
HA and TRN & $1 \mathrm{~m}$ & $1: 100$ & $10 \mathrm{~mm}$ \\
\hline
\end{tabular}

Table 3. Parameters of KUKA system (man (2004b) man (2004a))

\begin{tabular}{lll}
\hline & KR 16 & rail \\
\hline payload & $16 \mathrm{~kg}$ & - \\
supplementary load & $30 \mathrm{~kg}$ & - \\
static repeatability & $\pm 0.1 \mathrm{~mm}$ & $\pm 0.02 \mathrm{~mm}$ \\
max velocity & $2 \mathrm{~m} / \mathrm{s}($ at TCP) & $1.47 \mathrm{~m} / \mathrm{s}$ \\
remote control frequency & $85 \mathrm{~Hz}$ & $85 \mathrm{~Hz}$ \\
\hline
\end{tabular}

within a velocity range from zero to $0.5 \mathrm{~m} / \mathrm{s}$ (see table 1). The navigation precision requirements (see section 2.1) transform into a laboratory measure of $2 \mathrm{~mm}$ minimum (see table 2). To sufficiently judge the sensor output, the accuracy of the dynamic system should be better than these values.

A broad spectrum of sensors shall be tested. According to do Carmo et al. (2008), Weinberg et al. (2007) and Astrium (2006), the maximum projected weight of flight models is less than $10 \mathrm{~kg}$. Breadboard models might be heavier, but can often be divided into elements. In such a case solely the sensor head would be placed on the positioning device.

After a survey of candidate robotic systems the KUKA KR 16 was chosen. It is installed on a KL 250/2 rail which rests on a vibrationally isolated basement. The systems parameters are shown in Table 3 . The payload is $16 \mathrm{~kg}$. A supplementary load may be placed on the arm and on the base of the robot. This allows to take on additional hardware like power supplies or computing hardware. The static repeatability of $\pm 0.1 \mathrm{~mm}$ is one order of magnitude better than the required navigation precision in laboratory scale. The maximum traverse velocity is $1.47 \mathrm{~m} / \mathrm{s}$.

Furthermore the KR 16 allows to be real-time controlled via Ethernet using the proprietary protocol RSI-XML. More details are given in section 6.

\section{TERRAIN MODELS}

Depending on the stage of lunar landing, the terrain models have to fulfill different requirements.

For the coasting phase in the descent orbit, several projects and studies (e.g. see Cheng and Ansar (2005)) foresee absolute navigation. The principle is to identify extracted landmarks from live-images of the lunar surface with the help of an on-board database. By combining the recognized landmarks with the optical parameters of the sensor, the navigation solution is determined. The terrain model must therefore be manufactured with a high accuracy to the ground truth to be in good agreement with the on-board map.

The resolution of the model is driven by the navigation requirements and scale but also from the available digital elevation models (DEM) and the manufacturing limits. As a guideline we aimed to achieve a resolution in the order of the repeatability of the robot system. First work has been conducted with a computer generated lunar terrain model 
Table 1. Simulation options for TRON (numbers from internal simulations)

\begin{tabular}{|c|c|c|c|c|c|c|}
\hline landing phase & scale & $v_{S C}[\mathrm{~m} / \mathrm{s}]$ & $v_{l a b}[\mathrm{~m} / \mathrm{s}]$ & $\begin{array}{l}\text { downrange for } 10 \\
\mathrm{~m} \text { terrain model }\end{array}$ & altitudes & simulation time (RT) \\
\hline $\mathrm{DO}$ & $1: 50000$ & 1660 const. & 0.032 & $500 \mathrm{~km}$ & $50 \mathrm{~km}-100 \mathrm{~km}$ & $312 \mathrm{~s}$ \\
\hline DO & $1: 25000$ & 1660 const. & 0.064 & $250 \mathrm{~km}$ & $25 \mathrm{~km}-50 \mathrm{~km}$ & $120 \mathrm{~s}$ \\
\hline $\mathrm{DO}$ & $1: 10000$ & 1660 const. & 0.16 & $100 \mathrm{~km}$ & $10 \mathrm{~km}-20 \mathrm{~km}$ & $60 \mathrm{~s}$ \\
\hline PD & $1: 5000$ & $600-1660$ & $0.12-0.33$ & $50 \mathrm{~km}$ & $5 \mathrm{~km}-10 \mathrm{~km}$ & $30 \mathrm{~s}$ (starting at PDI) \\
\hline $\mathrm{PD}$ & $1: 2500$ & $150-600$ & $0.06-0.24$ & $25 \mathrm{~km}$ & $2.5 \mathrm{~km}-5 \mathrm{~km}$ & $60 \mathrm{~s}$ (starting at $5 \mathrm{~km}$ ) \\
\hline HA and TRN & $1: 100$ & $0-50$ & $0-0.5$ & $200 \mathrm{~m}$ lateral & $1000 \mathrm{~m}-100 \mathrm{~m}$ & dep. on hover time \\
\hline
\end{tabular}

to find suitable manufacturing parameters. The model was generated in a resolution of $2000 \times 2000$ pixels by using the software PANGU (Parkes et al. (2004)).

Due to the high accuracy requirements we chose milling for the manufacturing process and a polyurethane based foam (SIKA Block) as material. SIKA Block is used for master forms and deep drawing. It possesses a low shininess which is consistent with soil appearance. As a drawback it contains immanent bubbles, their size depending on the density of the material. The milling process is able to handle a material thickness of $10 \mathrm{~cm}$, which allows to represent altitude spans of $5000 \mathrm{~m}$ at 1:50000 and $1000 \mathrm{~m}$ at $1: 10000$.

At first a series of three test models, each of $0.5 \times 0.5$ $m^{2}$, had been milled at one constant milling resolution of $0.5 \mathrm{~mm}$ but different foam densities. Additionally the models were coated with a gray color. The highest density material (SIKA Block 610) was chosen. It possesses the smallest material-immanent bubbles and was the only sample where a smooth continuous coating was possible. Unfortunately the coating caused a total reflexion at low viewing angles. We continued the parameter finding process with the production of a fourth model. This time the focus was put on finding the right coating technique and milling resolution.

Therefore the model was coated and milled section-wise. A coating made of a mix of clear coat and matte-powder proved to overcome the total reflexion problem. At the same time this technique allows to put the desired color on the terrain. The milling was performed at different resolutions of $0.3 \mathrm{~mm} 0.2 \mathrm{~mm}$ and $0.1 \mathrm{~mm}$. Although 0.1 $\mathrm{mm}$ achieves the best surface quality, it also causes the highest cost and production time. To detect a detail of $0.2 \mathrm{~mm}$ in a distance of $1 \mathrm{~m}$, a mega-pixel camera needs a field of view (FOV) of $11.7^{\circ}$. So far in literature the sensors have much wider FOVs. Thus it was decided in favor to lower cost and production times and a milling resolution of $0.2 \mathrm{~mm}$ was chosen.

The lighting tests described in section 5 (Figure 3), show a good congruence between the digital elevation model and the milled model. Thus we are confident to have found a good solution for the production of the first terrain model. Currently we are processing data of the DEM data of Kaguya (Selene) mission, which have been recently published to the free accessible Selene Data Archive (Kag (2009)). The resolution of the data is $10 \mathrm{~m}$ horizontal and vertical. Such a resolution is sufficient to define a terrain model with a scale of 1:50000 and a milling resolution of 0.2 $\mathrm{mm}$. The projected size of the first terrain model based on Kaguya data is $3 \mathrm{~m}$ in width and $1 \mathrm{~m}$ in height, resulting in a data volume of $15000 \times 5000$ points. So far a $0.5 \times$
$0.5 \mathrm{~m}^{2}$ preview model has been manufactured to evaluate the quality of the Kaguya data. A comparison between a rendering of the DEM and the sunlit manufactured model showed satisfactory results. We expect to finish production of the full model by the end of August 2010.

The models for the powered descent could be manufactured in the same way as for the descent orbit. As described in section 2.3, scales from 1:2500 up to $1: 10000$ are candidate to simulate this orbit section. Considering a milling resolution of $0.2 \mathrm{~mm}$ the necessary DEM resolution is between $0.5 \mathrm{~m}$ and $2 \mathrm{~m}$. Unfortunately the Kaguya DEMs are limited to $10 \mathrm{~m} /$ pix. For milling, this leaves the option to wait for higher resolution data or to interpolate the dataset. Interpolation is not preferred because the resolution is increased only virtually, producing a smooth terrain without adding new details. As a consequence feature tracking algorithms might achieve less accurate results in comparison to non-interpolated data. To overcome this problem a different production approach of handmade models made of synthetic fibers is being explored. On the one hand is a low manufacturing precision, which is a drawback for absolute navigation purposes. On the other hand a big advantage is the very high level of detail (practically infinite) due to the manufacturing process. Although the details are of a random nature, this will impose no restriction for sensors which utilize an algorithm which is based on the tracking of unknown features, e.g. optical flow algorithms. If an absolute navigation had to be tested in PD, and a sufficient lunar DEM is not available, the hand-made model could be entirely laser-scanned with in-house equipment. The scan data would then serve as the ground truth. This option will be further explored in the upcoming work.

In the last phase of landing of many mission profiles (see section 2.1), a 3D model of the landing site is created on-board during the descent. As the lander gets in closer distance to the ground, this model is improving. The model provides the data for two methods. At first an absolute navigation is performed via the comparison of a premission on-board DEM with the live-DEM. This allows to determine the landing dispersion. Secondly, when reaching a sufficient resolution a safe landing site can be selected. A suitable scale for the last landing phase is 1:100. By using the milling process, which has a $10 \mathrm{~cm}$ vertical limit (see above in this section), an altitude range of $10 \mathrm{~m}$ could be simulated. But is very probable to experience a more dynamic terrain during the landing. For this reason we prefer the hand-made terrain model option, which do not possess the material thickness limit.

Once installed, the terrain models will be equipped with 
reflector targets of a laser scanner system. In this way the positions of the models with respect to the origin of the dynamic system can be determined in a precision of the order of $0.1 \mathrm{~mm}$.

\section{OPTICAL ENVIRONMENT}

The task of the lighting system is to provide the lighting conditions of the lunar environment. For future applications it shall also be able to simulate Mars or asteroid conditions. Due to the different distances to the sun, the lighting has to be variable in its intensity. Furthermore it should provide a near parallel lighting. During the landing manoeuvre the solar elevation angle might change. This is especially the case during the descent orbit, where orbit angles of up to $16^{\circ}$ are covered, which may cause a sun elevation change of up to $16^{\circ}$. Thus the lighting system must be able to change its elevation with respect to the terrain models.

For these reasons we are designing a 4-DOF dynamic system with the light source as payload. The DOFs are illustrated in Figure 1. The light will be installed on a movable frame which is connected to the robot to follow its traverse motion. During the motion along the terrain models the lights elevation can be changed by altering its position on the frame in combination with a rotation. In the same time the light can also be moved vertically. Several independently controllable actuators are foreseen for this task. The particular attitude of the light will be calculated by the transformation of the true sun position to the laboratory coordinates (see section 6).

First tests have been conducted with a zoom profile spotlight from ADB. It possesses a color temperature of 6000 $\mathrm{K}$ and can be dimmed by a shutter system in front of the aperture. The tests have been conducted in the laboratory, where the terrain test model 4 (see section 4) has been illuminated from a distance of $2 \mathrm{~m}$ and an elevation angle of $\approx 20^{\circ}$. For a comparison the same model has been exposed to sun light at approximately the same angle. A third measurement has been conducted via the rendering of this particular DEM in PANGU. The results are shown in Figure 3. They show a good congruence between each other. Some problems have been introduced by the mechanical shutter. Due to its principle of using multiple blades shadows tend to become non-uniform at shadow lengths $>0.2 \mathrm{~m}$. In the near future an approach to dim the light using an iris to overcome this problem will be tested.

The ambient lighting on bodies with no atmosphere is very low. On the Moon the main source of secondary light are reflections from the terrain, which leaves any shadows practically deep black. In the laboratory the situation is different. Once the light is being reflected by the terrain models it is multiple-reflected by the laboratory walls and creating an ambient light in the room. This causes the shadows on the terrain models to take on a gray color, instead of the desired deep black. This phenomenon is counteracted by wall-coverage with black fabric and ground-coverage with black carpet.

\section{HARDWARE-IN-THE-LOOP TEST CONCEPT}

In order to achieve a hardware-in-the-loop test for an optical navigation sensor the simulation of the environment
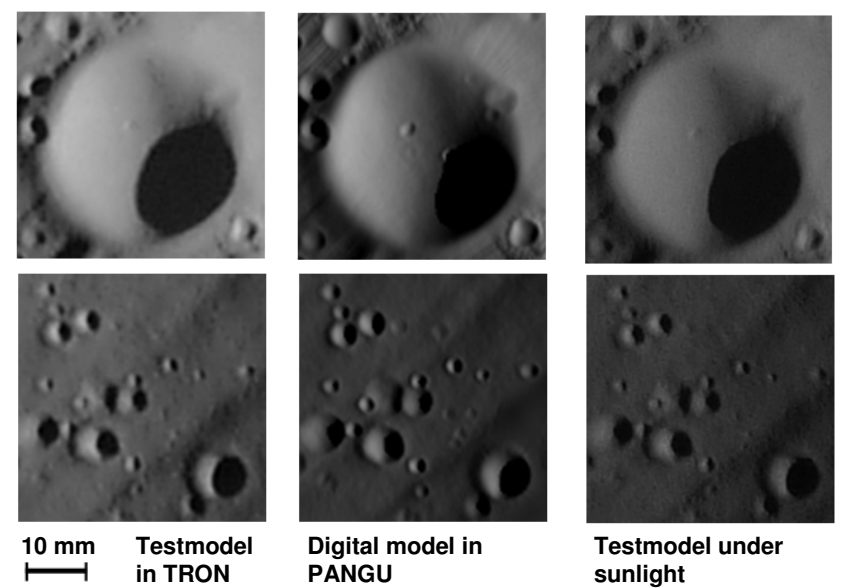

Fig. 3. Lighting tests with ADB light, PANGU, and sunlight; for all: elevation of illumination source $\approx$ $20^{\circ}$ from the right

and dynamics has to be controlled in real time. For that purpose the TRON facility uses a modular concept which is based on the real time simulation system dSPACE. On dSPACE compiled MATLAB/Simulink programs are executed. Figure 4 shows a schematic view of the concept. The concept allows to simulate the environment, vehicle dynamics, other navigation sensors, the actuation system as well as the on-board GNC computer (OBC) in real-time. For HiLT the optical navigation sensor is put in the loop as a real hardware. It is stimulated by the robot system and the lighting system.

For more complex system-wide tests other modules could be replaced by real hardware as well. For example the GNC computer emulation could be replaced by the real OBC hardware and the corresponding interfaces to the dSPACE simulator. The same could be done for the other navigation sensors like IMU or star tracker. The simulation models would be replaced by the hardware and the corresponding stimulator. The simulation would then additionally need a control module for the stimulator whereas the output of the sensor would be directly connected to the OBC hardware.

The control of the lab equipment consisting of the lighting system and the robot for motion simulation is done using three main modules. The first converts the simulated coordinates of position, attitude and their derivatives in the coordinate frame of the lab. These values are used to control the robot and to steer the lighting system in order to create the proper motion of the optical sensor with respect to the target model as well as to simulate the proper environmental (lighting) conditions.

The commanding of the robot is done via an Ethernet interface (dSPACE hardware DS 4504) which sends the corresponding RSI-XML commands. The command frequency is $85 \mathrm{~Hz}$. In our tests, the dSPACE system proved to provide a reliable $85 \mathrm{~Hz}$ Ethernet communication between the simulation and the robot controller.

\section{FUTURE WORK}

In this paper the state of TRON in June 2010 has been presented. It is illustrated in Figure 5. A full functionality of the descent orbit test shall be achieved by the end 


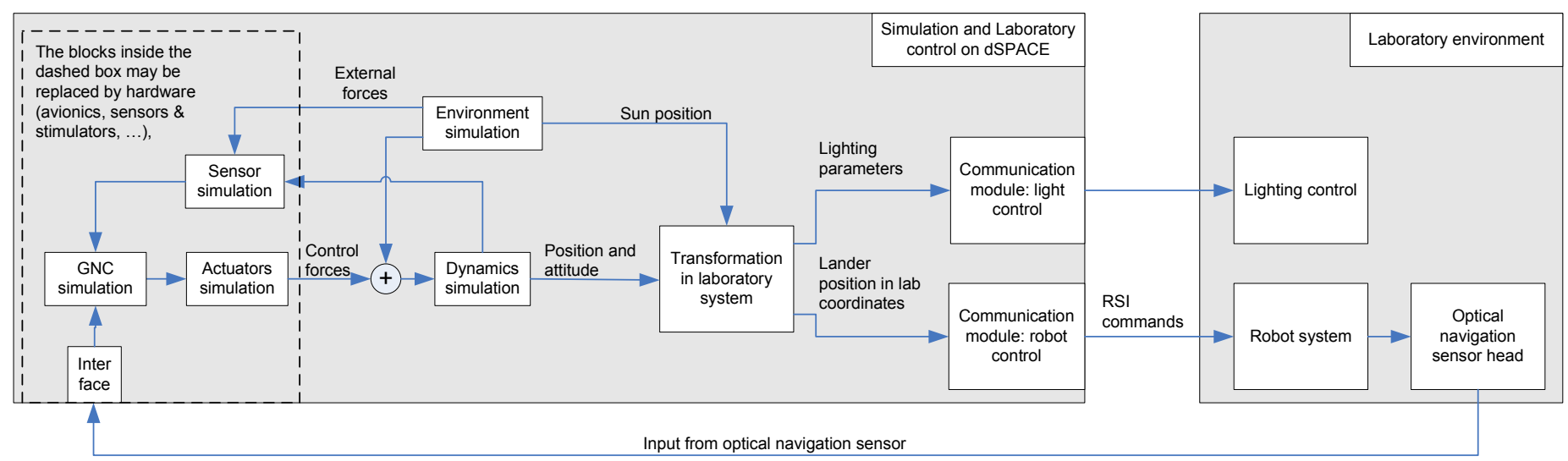

Fig. 4. Hardware-in-the-Loop test concept

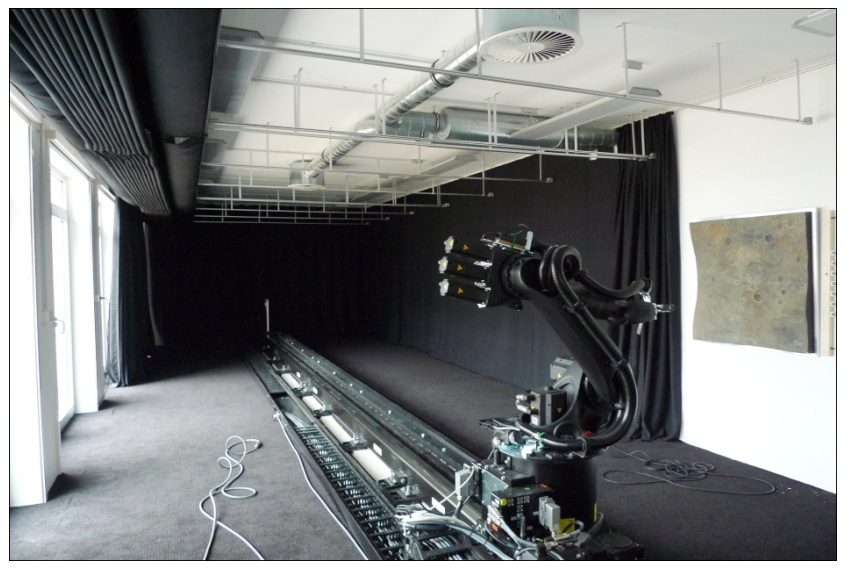

Fig. 5. Current status of TRON

of this year. The future work will therefore cover the production of a first lunar terrain model. Concerning the dynamic system, the necessary simulations will be installed on the dSPACE system, as well as the modules for the dynamic and lighting system. Furthermore the design and installation of the lighting system is envisaged.

The plan is to enhance the capability of TRON by the installation of an asteroid model. It will be equipped with a mechanism for rotation around its vertical axis. The maximum size is in the order of $4 \mathrm{~m}$ width, $4 \mathrm{~m}$ length and $1.5 \mathrm{~m}$ height.

\section{REFERENCES}

(2004a). Technical Data KL 250/2 Floor. KUKA Roboter $\mathrm{GmbH}$.

(2004b). Technical Data KR 16. KUKA Roboter GmbH. (2009). Selene data archive. URL https://www.soac.selene.isas.jaxa.jp/archive/ index.html.en.

Astrium, E. (2006). Navigation for planetary approach and landing - final report. Technical Report ESA Contract Reference 15618/01/NL/FM, ESA.

Cheng, Y. and Ansar, A. (2005). Landmark based position estimation for pinpoint landing on mars. In Robotics and Automation, 2005. ICRA 2005. Proceedings of the 2005 IEEE International Conference on, 4470-4475.

do Carmo, J.P., Moebius, B., Pfennigbauer, M. Bond, R., Bakalski, I., Foster, M., Bellis, S.,
Humphries, M., Fisackerly, R., and Houdou, B. (2008). Imaging lidars for space applications. volume 7061, 70610J. SPIE. doi:10.1117/12.793701. URL http://link.aip.org/link/?PSI/7061/70610J/1.

Houdou, B. (2008). Next Lunar Lander, Phase A Mission Study, Mission Requirements Document. Technical Report NEXT-LL-MRD-ESA(HME)-0001, ESA.

Loucks, M., Carrico, J., Carrico, T., and Deiterich, C. (2005). A comparison of lunar landing trajectory strategies using numerical simulations. Technical report, Analytical Graphics, Inc.

Neveu, D., Hamel, J.F., Christy, J., de Lafontaine, J., and Bilodeau, V. (2010). Next lunar lander: Descent \& landing gnc analysis, design and simulations. In Paper AAS 10-065 Guidance and Control Conference, Breckenridge, $C O$.

Parkes, S., Martin, I., and Dunstan, M. (2004). Planet surface simulation with PANGU. In Eighth International Conference on Space Operations, Montreal, Canada.

Sostaric, R. (2007). Powered descent trajectory guidance and some considerations for human lunar landing. In 30th Annual AAS Guidance And Control Conference.

Weinberg, J.D., Craig, R., Earhart, P., Gravseth, I., and Miller, K.L. (2007). Flash LIDAR Systems for Hazard Detection, Surface Navigation and Autonomous Rendezvous and Docking. LPI Contributions, 1371, 3023-+. Weinberg, J.D., Dissly, R., Nicks, D., and Miller, K.L. (2009). Applications and field testing of a flash lidar system for future planetary missions. In Lunar and Planetary Institute Science Conference Abstracts, volume 40 of Lunar and Planetary Inst. Technical Report, $2078-+$. 